\title{
PÓRTICOS METÁLICOS: CRITERIOS DE CÁLCULO
}

(METALLIC FRAMES: DESIGN CRITERIA)

José Monfort Lleonart

E.T.S. de Arquitectura de Valencia

ESPAÑA
Fecha de recepción: 21-IV-95

$430-5$

\section{RESUMEN}

Los pórticos metálicos, cuando carecen de elementos de estabilidad horizontal, resultan bastante sensibles a este tipo de desplazamientos, tanto cuando son debidos a las acciones exteriores como a sus propias imperfecciones geométricas.

Siguiendo los criterios del Eurocódigo 3, se expone el procedimiento de comprobación de estos elementos, tanto si disponen de arriostramiento como si no lo tienen, considerando la influencia de este efecto.

\section{SUMMARY}

When metallic frames lack horizontal stability elements, they become quite sensitive to horizontal displacements, both those produced by exterior actions and the ones due to their own geometric imperfections.

Acting on the criteria of Eurocode 3, the article brings the testing procedure for these elements, with or without bracing whose influence is taken into consideration.

\section{Generalidades}

Los pórticos son conjuntos de barras enlazadas entre sí, que forman un subconjunto estructural dentro de la estructura general de un edificio. Aunque normalmente se analizan como si fueran elementos planos independientes unos de otros, globalmente deben formar una estructura espacial que sea capaz de asegurar la estabilidad del edificio en cualquier dirección.

A partir de ahora nos referiremos a ellos como elementos planosy aislados, pero admitiendoque existen los elementos de arriostramiento necesarios para que se cumpla la condición expresada en el párrafo anterior.

Esquemáticamente, los tipos más frecuentes se indican en la figura 1, donde los casos (a) y (b) son característicos de construcciones de tipo industrial, y los (c) y (d), de los edificios de varias plantas.
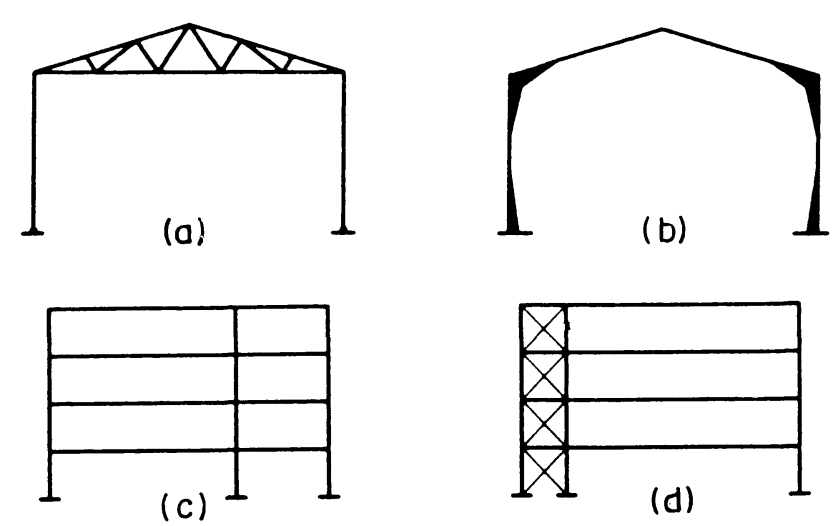

Fig. 1

Esta diferencia, además del aspecto meramente formal, se manifiesta también en el hecho de que en los edificios de varias plantas existen forjados entre los pórticos, que normalmente son elementos lo suficientemente rígidos como para enlazarlos y asegurar su comportamiento 
espacial, mientras que en las construcciones industriales no existen estos forjados, y se deben proyectar elementos estructurales específicos para realizar la función de enlace.

Básicamente están formados por:

-Vigas

Son elementos horizontales, o con inclinación relativamente pequeña, que trabajan principalmente a flexión, aunque pueden existir axiles, normalmente debidos a los empujes.

-Soportes

Son elementos verticales, que trabajan a compresión simple en el caso de que las uniones sean articuladas (salvo los momentos debidos a las excentricidades propias de los nudos, y a las acciones horizontales), y a flexocompresión en el caso de uniones rígidas.

\section{-Uniones}

Están constituidas por los enlaces entre vigas y soportes; pueden ser articuladas o rígidas (eventualmente semirrígidas).

\section{-Arriostramientos}

Son elementos que se colocan, algunas veces, para absorber los empujes horizontales.

Las formas características de colapso de los pórticos, las podemos clasificar en:
. colapso parcial

. colapso global o de conjunto

El colapso parcial, que corresponde al de alguno, o algunos, de los elementos que componen el pórtico, no suele llevar necesariamente a la ruina de la estructura completa, ya que en las estructuras hiperestáticas existen muchas formas posibles para la transmisión de las cargas hasta la cimentación, y en las isostáticas las barras son "casi independientes" unas de otras; en la figura 2 (a y b), se indican dos formas características de este tipo de colapso, que puede producirse.

\section{- En vigas:}

por formación de rótulas plásticas, inestabilidad lateral o abollamiento

\section{- En soportes:}

por pandeo en cualquiera de sus formas posibles, generalmente con consecuencias muy graves

\section{- En uniones:}

provocando la pérdida de equilibrio en el elemento que unen (si la rotura de la unión es completa), oporque cambia el comportamiento de la unión con respecto al que hemos considerado en el análisis de la estructura (si su rotura es incompleta).

El colapso global de la estructura corresponde a la pérdida de estabilidad de todo el conjunto simultáneamente; en la figura 2 (c) se indica un caso característico.

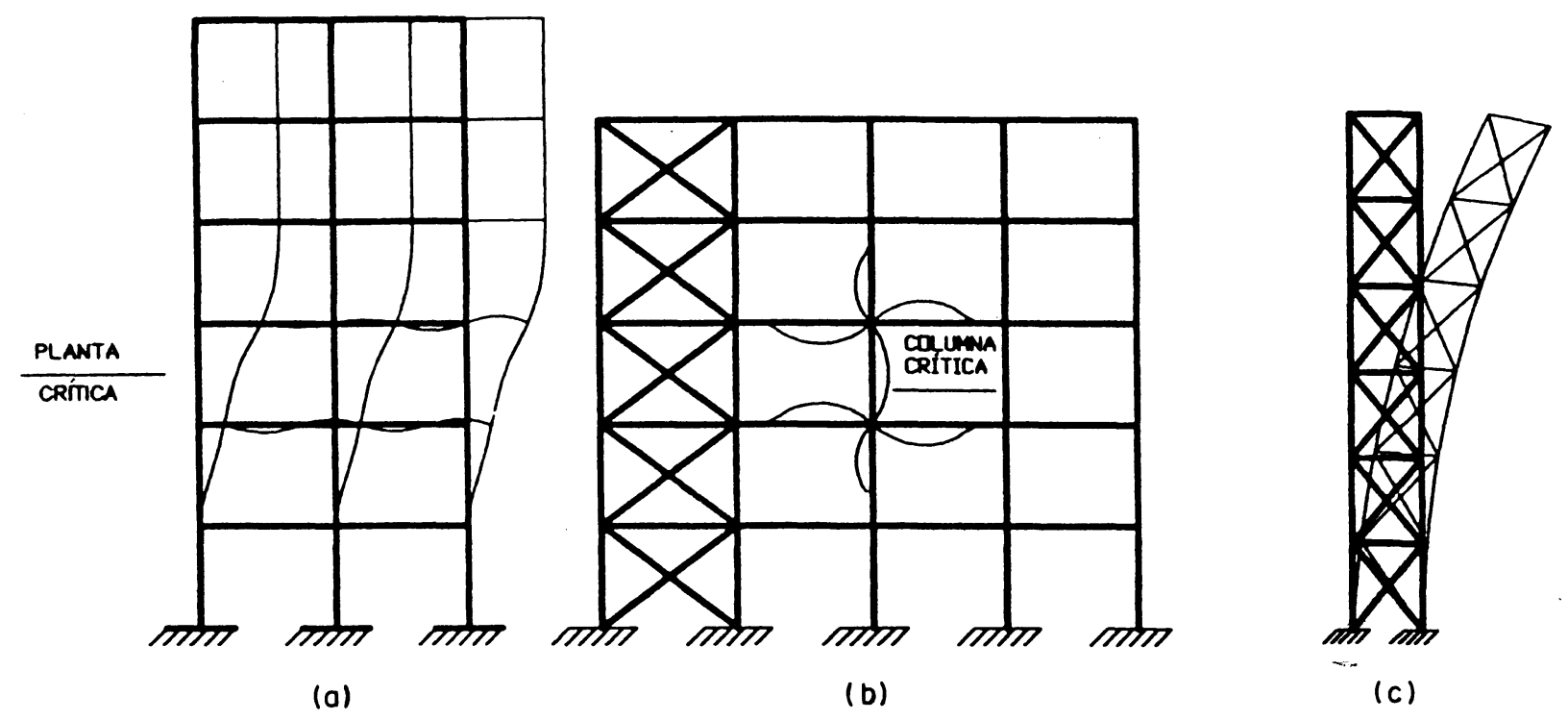




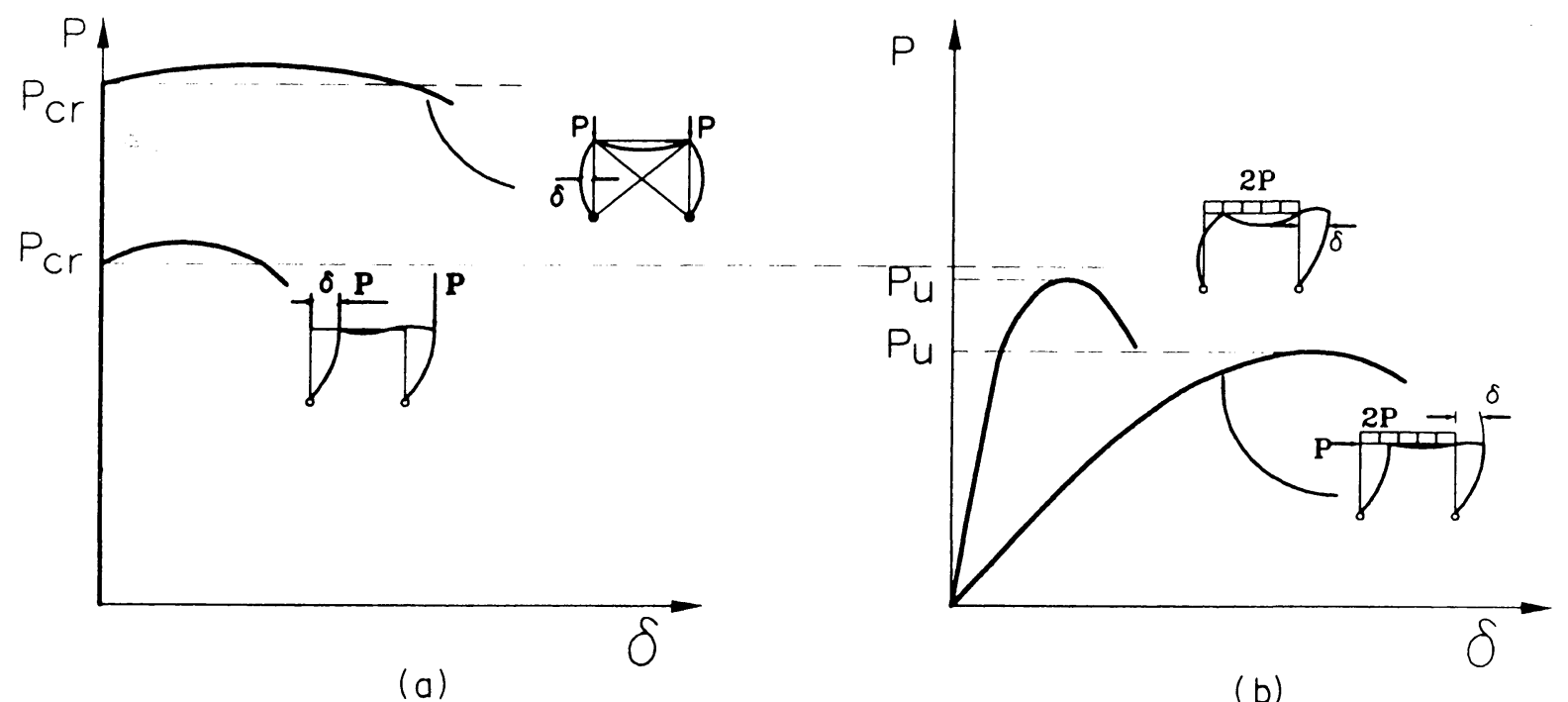

Fig. 3

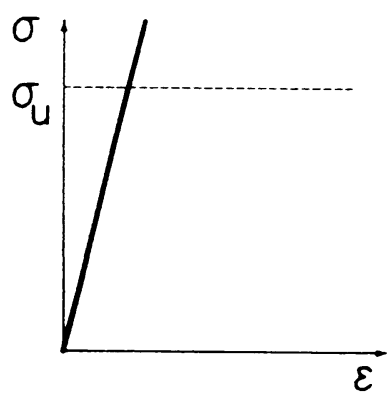

(a)

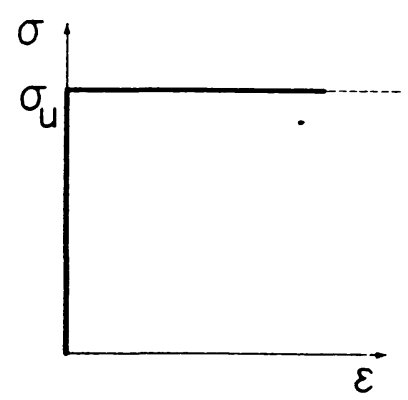

(b)

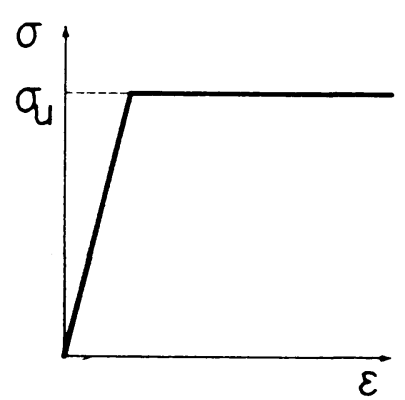

(c)
Desde el punto de vista de la relación entre fuerzas y desplazamientos, se produce esta situación de colapso global, cuando a un incremento pequeño de las cargas le corresponde un incremento grande de los desplazamientos; el valor de las cargas para el que se produce esta situación, se denomina carga crítica del pórtico (elástica o plástica, según el tipo de análisis que estemos efectuando). En la figura 3 (a y b) se presentan las gráficas correspondientes al diagrama cargas-desplazamientos cuando se produce esta situación, según exista o no flexión desde el principio de la actuación de las cargas, respectivamente.

\section{Métodos de análisis}

Estos métodos los podemos clasificar desde dos puntos de vista, que a su vez los podemos combinar entre ellos:

-por el tipo de diagrama simplificado de tensióndeformación que adoptemos para el material; se suele considerar, diagrama elástico, rígido-plástico, o elastoplástico, figura 4 (a, b y c), respectivamente.

-por la forma de considerar la influencia de las deformaciones sobre las acciones que actúan sobre la estructura: si despreciamos la influencia de estas deformaciones, y planteamos las condiciones de equilibrio sobre la configuración inicial (no deformada) de la estructura, se trata de análisis de primer orden; si tenemos en cuenta que las deformaciones debidas a las acciones exteriores modifican el efecto de estas acciones, y planteamos las condiciones de equilibrio sobre la configuración deformada de la estructura, estaremos realizando análisis de segundo orden, donde, a su vez, podemos distinguir entre los efectos de segundo orden debidos a los desplazamientos de conjunto de la estructura, y los debidos a las deformaciones locales de las barras, figura 5 .

Cuando se deba tener en cuenta la influencia de los desplazamientos, habrá que considerar también que las 


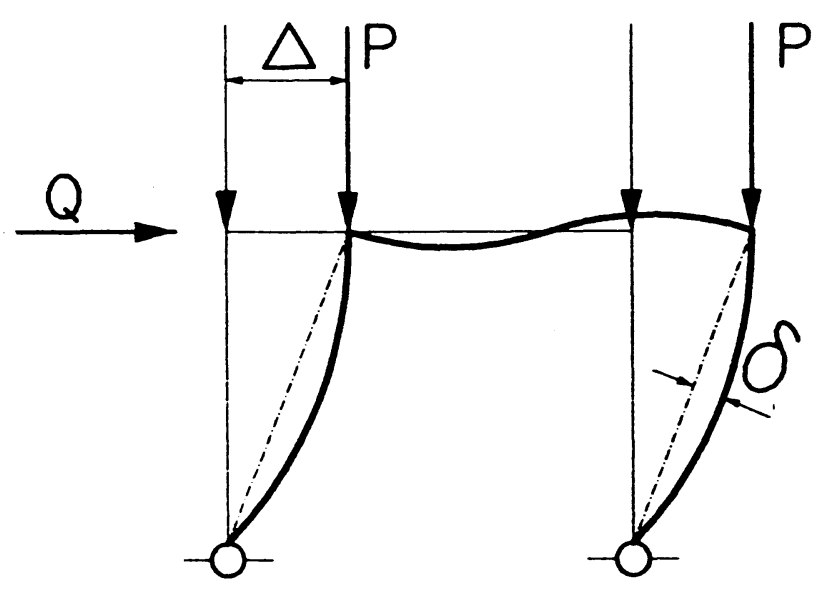

P. $\Delta$ momento de segundo orden debido a desplazamientos de conjunto de la estructura.

P. $\delta$ momento de segundo orden debido a deformaciones particulares de las columnas.

Fig. 5

estructuras reales tienen imperfecciones geométricas, que influyen sobre los efectos de las acciones de forma similar a los desplazamientos de la estructura, por lo que también habrá que considerarlas; en el epígrafe siguiente se dan criterios para estimar el valor de estas imperfecciones.
Combinando los dos aspectos anteriores, podemos realizar los tipos de análisis que se indican en la figura 6; como puede verse, a medida que el procedimiento de análisis es más completo, nos aproximamos más al comportamiento real de la estructura. Todas estas gráficas corresponden al caso en que el fallo se produce por inestabilidad global, sin considerar que se produzcan efectos de abollamiento, pandeo de alguna barra ofalta de capacidad de rotación en las rótulas plásticas que se forman sucesivamente. En ellas tenemos:

\section{Análisis elástico de primer orden}

Consideramos para el material un comportamiento elástico indefinido, y las condiciones de equilibrio se plantean sobre la geometría no deformada; la gráfica, en este caso, es una recta que llega hasta la carga crítica elástica (corresponde a la bifurcación de equilibrio calculada sin considerar la influencia de los desplazamientos sobre las acciones exteriores).

Los resultados así obtenidos sólo son aproximados en el tramo inferior de la gráfica.

\section{Análisis elástico de segundo orden}

Se considera también que el comportamiento del material es elástico indefinido, pero las ecuaciones de equilibrio se plantean en la geometría deformada; los desplazamientos

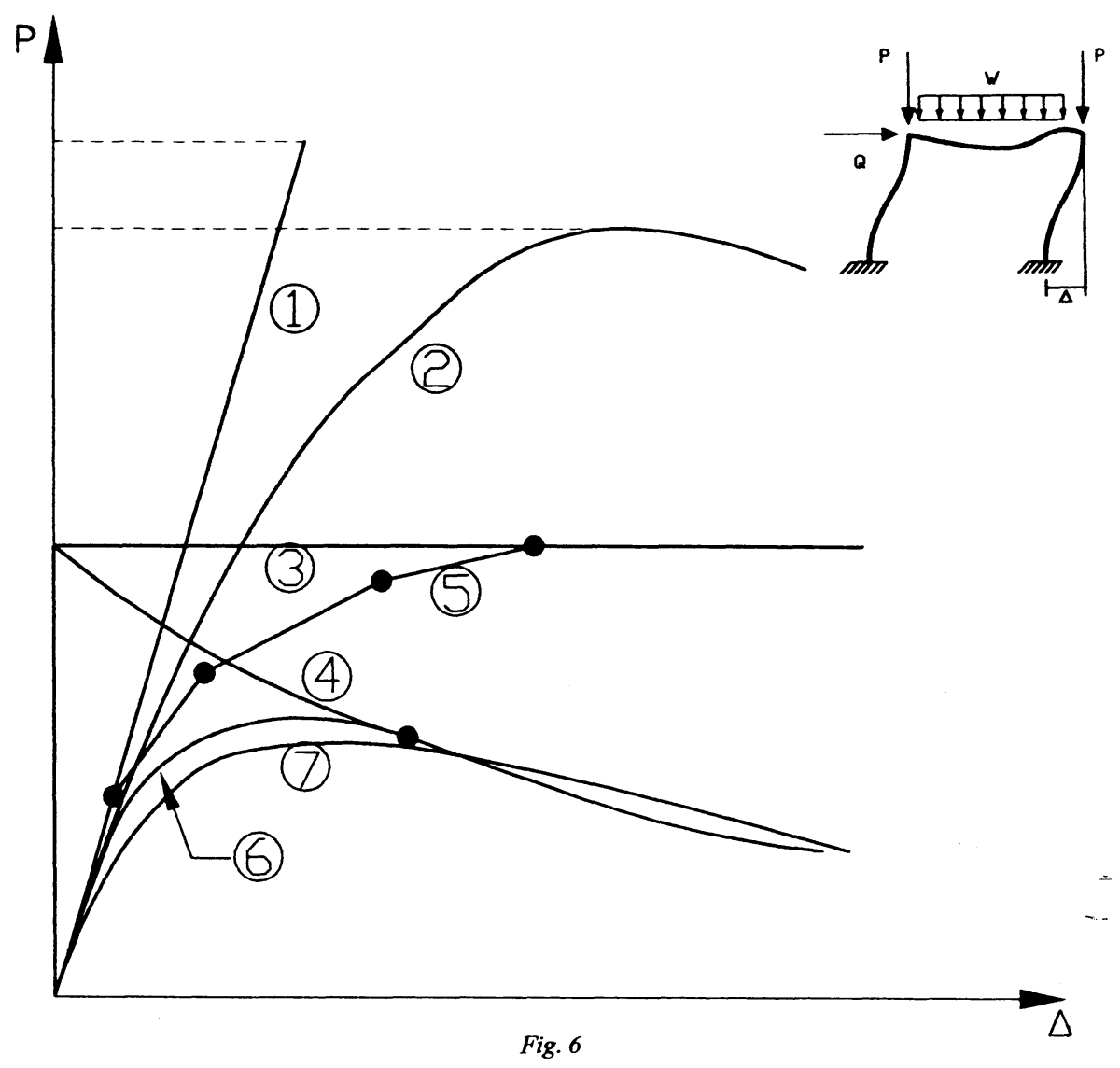


crecen más rápidamente, por la no linealidad geométrica, lo que hace que la gráfica sea una curva, que alcanza un máximo que corresponde a la carga elástica de rotura (a partir de ella sólo es posible el equilibrio si la carga disminuye).

\section{Análisis rígido-plástico de primer orden}

Si consideramos un comportamiento rígido-plástico del material, y planteamos las condiciones de equilibrio en la geometría no deformada, los desplazamientos serán nulos hastaque las acciones exteriores alcancen el valor necesario para originar la aparición de un número suficiente de rotulas plásticas que haga que la estructura, o una parte de ella, se convierta en un mecanismo.

A partir de aquí, el material tiene un comportamiento plástico y las deformaciones crecen de forma ilimitada para ese valor constante de la carga.

\section{Análisis rígido-plástico de segundo orden}

Corresponde a la misma situación anterior, pero ahora planteamos las condiciones de equilibrio en la geometría deformada; hasta la transformación de la estructura en un mecanismo, el comportamiento es idéntico (corresponde a la zona rígida del material), pero a partir de aquí, sólo es posible el equilibrio si la carga disminuye (por la no linealidad geométrica).

\section{Análisis elasto-plástico de primer orden}

Planteamos las condiciones de equilibrio en la geometría no deformada y realizamos una serie de análisis elásticos sucesivos después de la aparición de cada una de las rótulas (cada vez la estructura es más deformable y cada tramo es una recta porque estamos considerando comportamiento elástico del material); cuando la estructura se transforma en un mecanismo, pasaa tener un comportamiento plástico, y el desplazamiento aumenta ilimitadamente bajo carga constante.

El tramo hasta la formación de la primera rótula, coincide con el correspondiente al análisis elástico de primer orden.

\section{Análisis elasto-plástico de segundo orden}

Es un proceso similar al anterior, pero planteando en cada etapa las condiciones de equilibrio en la geometría deformada.

Cada tramo es ahora una curva por la no linealidad geométrica, el primero coincide sensiblemente con el correspondiente al análisis elástico de segundo orden, y el tramo final coincide con la gráfica 4, puesto que en ambos casos la estructura se ha transformado en un mecanismo y tiene un comportamiento plástico.
A partir de un valor dado de las acciones exteriores, sólo es posible el equilibrio si se produce una disminución de esta carga.

\section{Comportamiento real}

Estos valores se suponen obtenidos experimentalmente.

\section{Imperfecciones}

Como se haindicado antes, para el estudio de cualquier tipo de estructura metálica, se deben tener en cuenta los efectos de las imperfecciones de las piezas y de los pórticos reales, considerando tanto las debidas a las tensiones residuales, como las imperfecciones geométricas (desplomes, excentricidades no previstas, falta de ajuste, en el supuesto de que sus valores son menores que las tolerancias admisibles).

Estas imperfecciones se tendrán en cuenta en:

. análisis global

. análisis de arriostramientos

. dimensionado de las piezas

y se aplicarán considerando unas deformaciones adicionales apropiadas, que se especifican a continuación para cada caso.

\subsection{Imperfecciones de los pórticos}

Todas sus imperfecciones se asimilan a una imperfección geométrica equivalente, representada por un desplazamiento $\phi$, figuras 7 (a) y 8 (a), dado por

siendo:

$$
\phi=\mathrm{k}_{\mathrm{c}} \cdot \mathrm{k}_{\mathrm{s}} \cdot \phi_{0}
$$

$$
\begin{aligned}
& \phi_{0}=1 / 200 \\
& \mathrm{k}_{\mathrm{c}}=\left[0,5+\frac{1}{\mathrm{n}_{\mathrm{c}}}\right]^{1 / 2} \leq 1,0 \\
& \mathrm{k}_{\mathrm{s}}=\left[0,2+\frac{1}{\mathrm{n}_{\mathrm{s}}}\right]^{1 / 2} \leq 1,0 \\
& \mathrm{n}_{\mathrm{c}}=\text { número de pilares en cada planta } \\
& \mathrm{n}_{\mathrm{s}}=\text { número de plantas }
\end{aligned}
$$

En el valor $n_{c}$ se incluirán sólo aquellos pilares que soporten una carga vertical $\mathrm{N}_{\text {sd }}$ de al menos el $50 \%$ del valor medio de la carga vertical por cada pilar en la planta correspondiente (en caso contrario, ài ser un pilar poco cargado, la influencia de las imperfecciones es muy pequeña). 


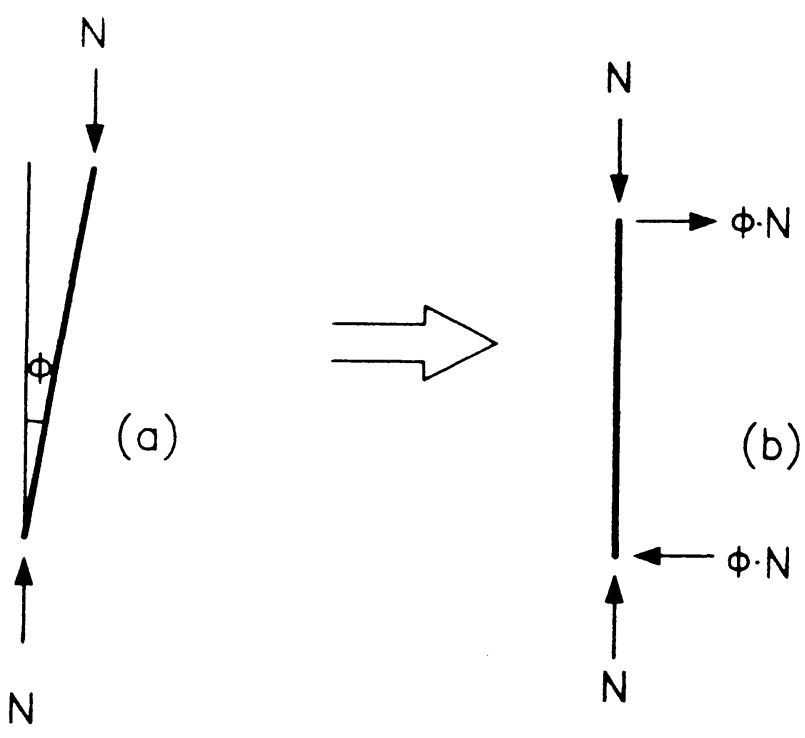

Fig. 7 figuras 9 y 10 (cuando haya varias combinaciones de valores que satisfagan esta condición, se podrá usar cualquiera de ellas).

Estos desplazamientos horizontales se pueden producir en cualquier dirección, pero se considerará unaúnica dirección cada vez; a efectos de torsión, si que deberá considerarse la posibilidad de desplazamientos en sentido contrario en dos caras opuestas, figura 11.

Estas imperfecciones, si seconsidera conveniente, se pueden sustituir por fuerzas horizontales equivalentes, que en el caso de pórticos de edificación, se aplicarán en cada planta y serán proporcionales a las cargas verticales que actúen en ella, figuras 7 (b) y 8 (b).

Las reacciones horizontales de cada soporte se calcularán utilizando las imperfecciones iniciales, y no los esfuerzos horizontales equivalentes; si no actúan cargas horizontales,
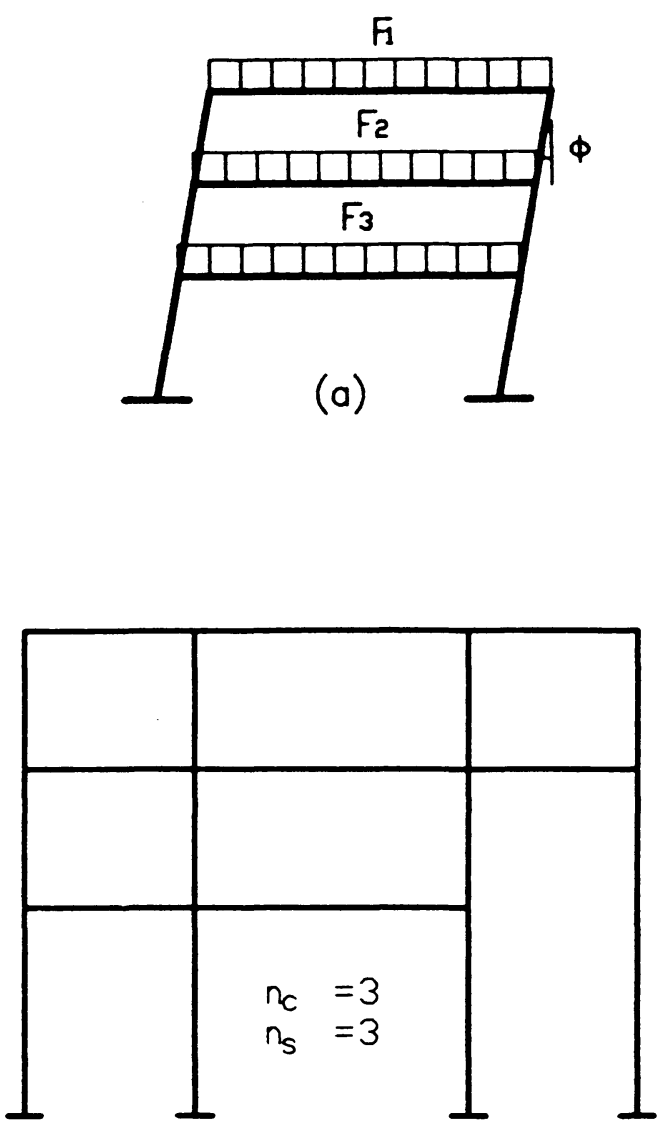

Fig. 9

Asimismo, en el valor de $n_{c}$ se incluirán sólo los pilares que se prolonguen en todas las plantas incluidas en $n_{s}$; en el valor de $n_{s}$ se incluirán únicamente aquellas plantas que estén conectadas con todos los soportes incluidos en $n_{c}$,

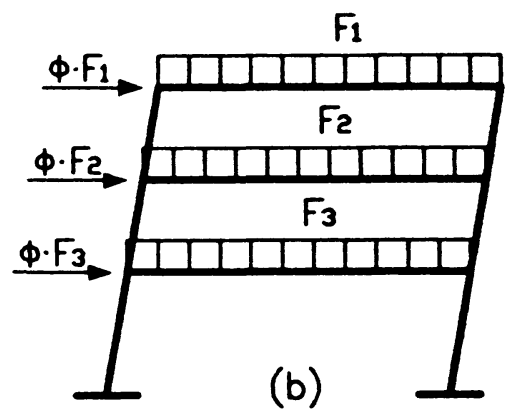

Fig. 8

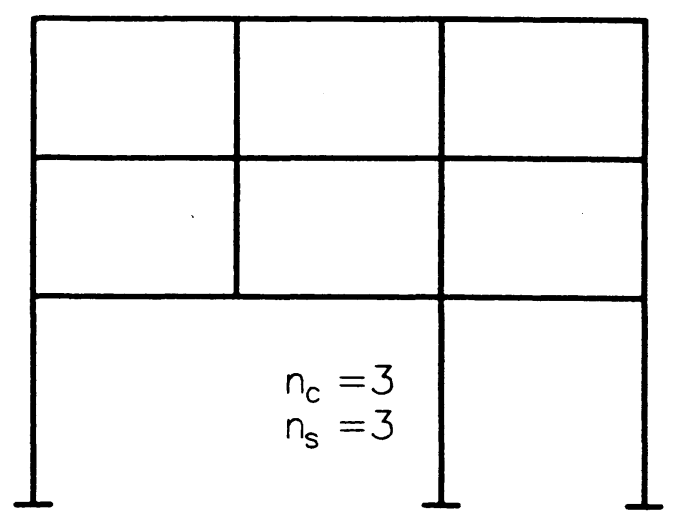

Fig. 10

la resultante horizontal de las reacciōnes en cada planta será cero (porque el efecto de las imperfecciones se traduce en un par de fuerzas en el soporte, y por tanto su resultante es nula, ver figura 7). 


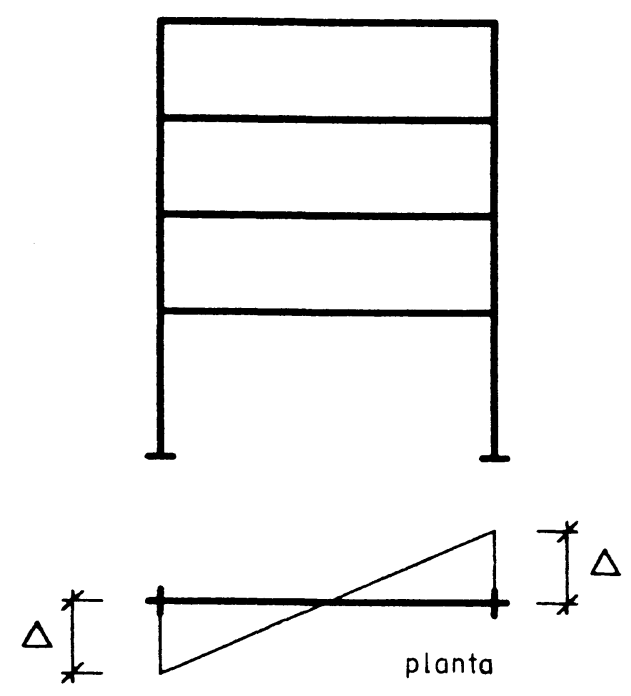

Fig. 11

Estos efectos de las imperfecciones se tendrán en cuenta en el análisis global de la estructura, y las solicitaciones que así resulten se considerarán, junto con las demás acciones para el dimensionado de los elementos.

\subsection{Imperfecciones en los sistemas de cubierta arriostrados}

Nos referiremos en este apartado a los elementos que se utilizan para estabilizar vigas o barras comprimidas (ejemplo, contravientos de construcciones industriales); la influencia de las imperfecciones en los elementos arriostrados, se considera también mediante una imperfección geométrica equivalente, representada por una excentricidad inicial $e_{0}$, figura 12 (a), de valor

donde:

$$
e_{0}=\frac{k_{r} \cdot L}{500}
$$

$$
\begin{aligned}
& \mathrm{L}=\text { longitud del elemento arriostrado } \\
& \mathrm{k}_{\mathrm{r}}=\left[0,2+\frac{1}{\mathrm{n}_{\mathrm{r}}}\right]^{1 / 2} \leq 1,0 \\
& \mathrm{n}_{\mathrm{r}}=\text { número de puntos que se arriostran }
\end{aligned}
$$

A efectos de cálculo, esta excentricidad inicial, se puede sustituir por un esfuerzo estabilizador equivalente, figura 12 (b), representado por una carga uniforme repartida, cuyo valor unitario q viene dado por:

Para una sola pieza arriostrada

$$
\begin{aligned}
& q=\frac{\mathrm{N}}{50 \cdot \mathrm{L}} \longrightarrow \text { si } \delta_{\mathrm{q}} \leq \frac{\mathrm{L}}{2.500} \\
& \mathrm{q}=\frac{\mathrm{N}}{60 \cdot \mathrm{L}} \cdot(1+\alpha) \longrightarrow \text { si } \delta_{\mathrm{q}}>\frac{\mathrm{L}}{2.500}
\end{aligned}
$$

siendo $\delta_{q}$ la flecha del arriostramiento en su plano debida a cualquier acción exterior que actúe sobre él, incluida la carga $\mathrm{q}, \mathrm{y}$

$$
\alpha=\frac{500 \cdot \delta_{q}}{L} \geq 0,2
$$

. Para múltiples piezas arriostradas

$$
\begin{aligned}
& \mathrm{q}=\frac{\Sigma \mathrm{N}}{60 \cdot \mathrm{L}} \cdot\left(\mathrm{k}_{\mathrm{r}}+0.2\right) \longrightarrow \text { si } \delta_{\mathrm{q}} \leq \frac{\mathrm{L}}{2.500} \\
& \mathrm{q}=\frac{\Sigma \mathrm{N}}{60 \cdot \mathrm{L}} \cdot\left(\mathrm{k}_{\mathrm{r}}+\alpha\right) \longrightarrow \text { si } \delta_{\mathrm{q}}>\frac{\mathrm{L}}{2.500}
\end{aligned}
$$

En ambos casos, cuando el elemento (o elementos) que se arriostra sea una viga, el esfuerzo $\mathrm{N}$ se adoptará

$$
\mathrm{N}=\mathrm{M} / \mathrm{h}
$$

siendo:

$$
\begin{aligned}
& \mathrm{M}=\text { momento máximo en la viga } \\
& \mathrm{h}=\text { canto total de la viga }
\end{aligned}
$$

En los puntos de empalme de las piezas comprimidas, se considerará sobre el arriostramiento un esfuerzo local adicional de valor

$$
k_{r} \cdot N / 100
$$

por cada elemento comprimidocon uniớn en ese punto, que apoya sobre el arriostramiento; a su vez,; el arriostramiento tiene que poder transmitir este esfuerzo a los puntos fijos de la pieza arriostrada, figura 13. 


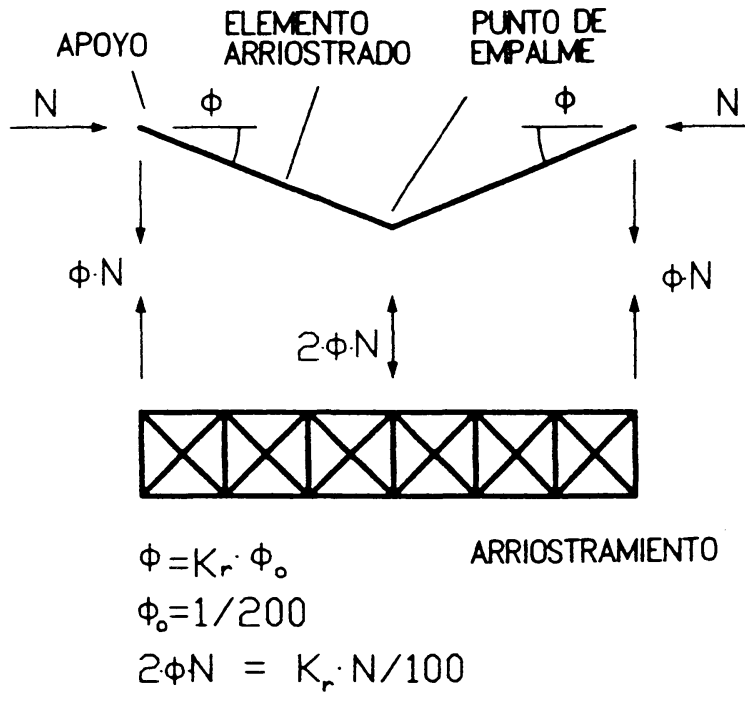

Fig. 13

Para la comprobación de este esfuerzo localizado, se considerará también sobre el arriostramiento cualquier acción exterior que actúe directamente sobre él (incluidas las de sus propias imperfecciones y las de sus apoyos), pero no será necesario considerar los esfuerzos debidos a las imperfecciones de los elementos arriostrados, a que se ha hecho referencia antes en este mismo epígrafe.

\subsection{Imperfecciones de las piezas}

Las imperfecciones de las piezas ya se tienen en cuenta en su dimensionado si se utilizan las Curvas Europeas de Pandeo, por lo que no es necesario considerarlas adicionalmente.

\section{Rigidez horizontal}

Todas las estructuras deben tener rigidez suficiente a los desplazamientos laterales, y esto puede conseguirse mediante:

- la rigidez propia de los pórticos (triangulaciones, nudos rígidos, pilares empotrados en su base o nudos semirrígidos, siempre que se compruebe su rigidez al giro); es decir, los elementos que proporcionan esta rigidez se considera que forman parte del pórtico.

. la rigidez de elementos de arriostramiento (celosías, pórticos rígidos, muros, núcleos, etc.); es decir, estos elementos se consideran ajenos al pórtico, sólo le proporcionan apoyo horizontal, y los sistemas se calculan independientemente.

En base a estos elementos se considera un pórtico intraslacional, cuando su respuesta a los esfuerzos

(c) Consejo Superior de Investigaciones Científicas Licencia Creative Commons 3.0 España (by-nc) horizontales contenidos en su plano es tal que sean despreciables las solicitaciones adicionales debidas a los desplazamientos horizontales de sus nudos.

En casocontrario, se considerará como pórtico traslacional, y en su dimensionado se considerarán los efectos de los desplazamientos horizontales.

Para precisar este criterio, los pórticos planos de edificación con vigas unidas a pilares en cada planta (cuadrícula completa) se considerarán como intraslacionales para un estado de cargas dado, si, cuando se utilice la teoría de primer orden, los desplazamientos horizontales en cada planta producidos por las cargas horizontales y verticales de cálculo, y a las imperfecciones debidas a su desplazamiento inicial aplicadas como esfuerzos equivalentes, verifican:

$$
\frac{\delta}{\mathrm{h}} \cdot \frac{\mathrm{V}}{\mathrm{H}} \leq 0,1
$$

siendo:

$\delta=$ desplazamiento horizontal relativo entre una planta y la superior

$\mathrm{h}=$ altura entre plantas

$\mathrm{H}=$ reacción horizontal total en la base de la planta

$\mathrm{V}=$ reacción vertical total en la base de la planta

\subsection{Elementos de arriostramiento}

Un pórtico metálico puede considerarse arriostrado cuando existe una estructura que tiene rigidez suficiente como para que podamos considerar que resiste todas las cargas horizontales; en la práctica, puede suponerse esto cuando el elemento de arriostramiento reduce los desplazamientos horizontales en al menos un $80 \%$, con respecto a los de la misma estructura sin arriostrar.

En estas condiciones, el pórtico arriostrado puede considerarse intraslacional; el elemento de arriostramiento, a su vez, podrá ser traslacional o intraslacional.

Todas las acciones horizontales, y los esfuerzos horizontales equivalentes a las imperfecciones, podremos considerar que actúan únicamente sobre el elementode arriostramiento, que lo deberemos dimensionar para resistir:

- todas las cargas horizontales que actúan sobre el pórtico que arriostra.

- todas las cargas horizontales $y / 0$ verticales que actúan sobre el propio arriostramiento (incluidas las acciones equivalentes producidas por sus propias imperfecciones).

- los efectos del desplazamiento inicial debido a las imperfecciones (o los esfuerzos horizontales equivalentes) de todos los pórticos que arriostra. 


\section{Estabilidad}

Todas las comprobaciones a realizar sobre los pórticos, deberán hacerse teniendo en cuenta su carácter traslacional o intraslacional.

Todos los pórticos, incluidos los traslacionales, deben también ser comprobados a rotura en modo intraslacional.

En el caso de pórticos intraslacionales, la influencia de los desplazamientos horizontales es despreciable, como se ha indicado en el apartado 4. En los traslacionales, las comprobaciones se efectuarán por uno de los procedimientos siguientes.

\subsection{Análisis elástico de pórticos traslacionales}

En el estudio global se tendrán en cuenta los efectos de segundo orden, bien directamente mediante un análisis elástico de segundo orden (considerando en el plano del pórtico las longitudes de pandeo correspondientes al estado intraslacional), o indirectamente, mediante una de las dos siguientes alternativas:

- análisis elástico de primer orden, con momentos amplificados como consecuencia del desplazamiento.

análisis elástico de primer orden, con las longitudes de pandeo correspondientes a la posición desplazada.

El primero de los procedimientos(momentos amplificados), no deberá utilizarse cuando la relación

$$
\frac{\mathrm{V}_{\mathrm{sd}}}{\mathrm{V}_{\mathrm{cr}}}>0,25
$$

siendo:

$\mathrm{V}_{\mathrm{sd}}=$ valor de cálculo de la carga vertical total

$\mathrm{V}_{\mathrm{cr}}=$ valor elástico crítico en el momento de la rotura en estado traslacional

En los pórticos de edifícación con cuadrícula completa, esta relación puede tomarse

$$
\frac{\mathrm{V}_{\mathrm{sd}}}{\mathrm{V}_{\mathrm{cr}}}=\frac{\delta}{\mathrm{h}} \cdot \frac{\mathrm{V}}{\mathrm{H}}
$$

con los mismos significados de estas magnitudes indicados anteriormente en [8].

Cuando sea aplicable este procedimiento, los momentos resultantes del análisis elástico de primer orden se amplificarán multiplicándolos por la relación

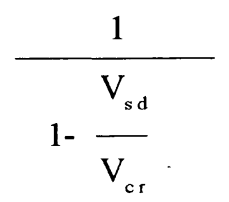

y para el dimensionado de las barras se tomarán las longitudes de pandeo en el plano del pórtico correspondientes al estado intraslacional.

Cuando se utilice el segundo planteamiento para el dimensionado de los soportes (análisis de primer orden con longitudes de pandeo correspondientes al estado traslacional), los momentos en las vigas, así como los de las uniones viga-soporte, deberán amplificarse al menos por un coeficiente 1,2 salvo que se demuestre que es adecuado utilizar un valor menor.

\subsection{Análisis plástico de pórticos traslacionales}

Para el análisis plástico global se tendrán en cuenta los efectos de segundo orden en estado traslacional; esto puede hacerse, directamente, mediante análisis elasto-plástico de segundo orden (en cuyo caso, deben disponerse arriostramientos laterales al pórtico en los puntos donde se puedan formar rótulas plásticas bajo cualquier estado de cargas posible), o bien mediante análisis rígido-plástico considerando los efectos de segundo orden indirectamente, en los casos que se indican a continuación:

1. Pórticos de una o dos alturas en los que

- no se producen rótulas plásticas en los pilares

- los pilares cumplen todas las prescripciones que se indican posteriormente

2. Pórticos empotrados en sus bases, en los que al comprobar la rotura en estado traslacional sólo se forman rótulas plásticas en sus bases, figura 14 , lo que equivale a efectuar

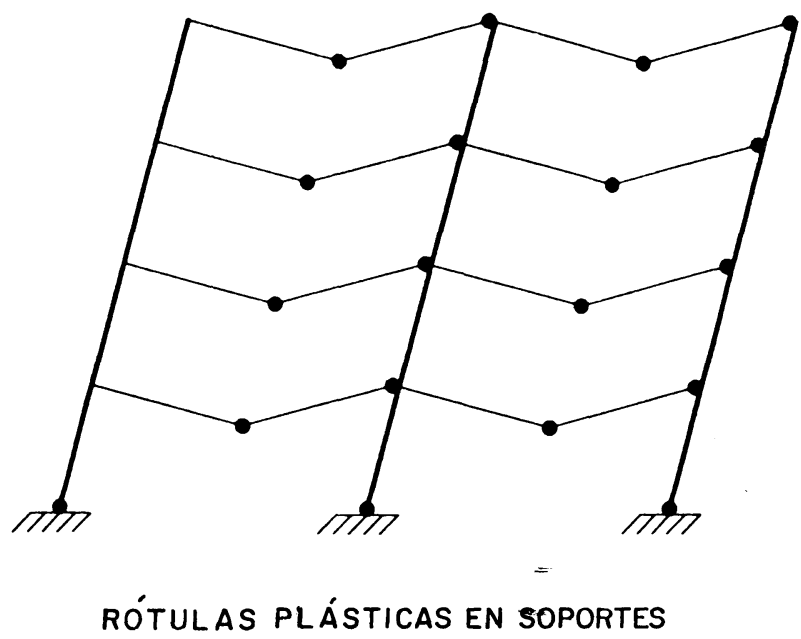

Fig. 14 
el dimensionado según un mecanismo incompleto, de forma que los pilares continúen en fase elástica cuando se alcance el momento necesario para la formación de rótulas plásticas

3. Cuandola relación indicada en [10] cumplala limitación

$$
\mathrm{V}_{\mathrm{sd}} / \mathrm{V}_{\mathrm{cr}} \leq 0,2
$$

amplificando todas las fuerzas internas y los momentos por la relación indicada en [11]

4. Para el dimensionado de la estructura se tomarán las longitudes de pandeo en el plano de la estructura correspondientes al estado intraslacional, y se determinarán teniendo en cuenta el efecto de las rótulas plásticas

5. En los pilares, cuando se formen rótulas, se comprobara que éstas tienen la capacidad de giro necesaria; no será necesario realizar esta comprobación en secciones Clase 1.

6. Cuando exista la posibilidad de formación de rótulas plásticas en pilares de pórticos diseñados mediante análisis rígido-plástico de primer orden, la esbeltez de estos pilares en el plano de la estructura deberá verificar
- en pórticos arriostrados

$$
\bar{\lambda} \leq 0,40 \cdot\left[\frac{\mathrm{A} \cdot \mathrm{f}_{\mathrm{y}}}{\mathrm{N}_{\mathrm{sd}}}\right]^{1 / 2}
$$

- en pórticos no arriostrados

$$
\bar{\lambda} \leq 0,32 \cdot\left[\frac{\mathrm{A} \cdot \mathrm{f}_{\mathrm{y}}}{\mathrm{N}_{\mathrm{sd}}}\right]^{1 / 2}
$$

donde $\bar{\lambda}$ es la esbeltez adimensional en el plano del pórtico calculada, tomando como longitud de pandeo la de la barra.

7. Salvo para el caso indicado en 2 , el análisis rígidoplástico de primer orden para la comprobación global, no debe utilizarse para pórticos sin arriostramiento de más de dos plantas.

\section{REFERENCIAS}

Eurocódigo 3. Estructuras de acero

Eurocódigo 4. Estructuras mixtas de acero-hormigón
Structural Eurocodes, IABSE Conference. Davos 1992

Estructuras de acero, O. López de Heredia. Edit. Limusa

\section{publicación del IETCC/CSIC}

\section{ACUEDUCTOS ROMANOS EN ESPAÑA \\ Carlos Fernández Casado}

Prof. Dr. Ing. de Caminos, Canales y Puertos

Esta publicación se compone de una serie de articulos, publicados en la Revista "Informes de la Construcción", en los cuales se hace un análisis de los acueductos romanos que existen en España y el balance de las condiciones de conservación en que se encuentra cada uno de ellos, incluyendo referencias históricas y literarias. Se ha ilustrado con la reproducción de la valiosa documentación gráfica que posee el prestigioso autor.

Un volumen encuadernado en couché, a dos colores, de $21 \times 27$ centimetros, compuesto de 238 páginas, numerosos grabados, dibujos, fotos en blanco y negro y figuras de línea.

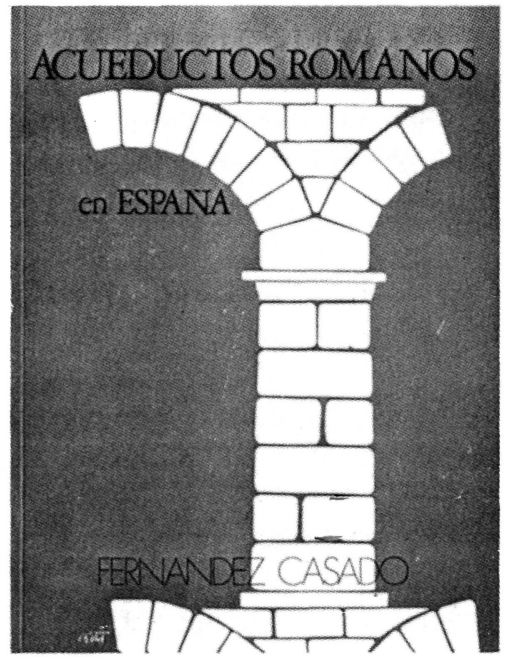

